\title{
MALARIA: PROPHYLAXIS IS A CORNERSTONE IN MANAGEMENT Short Communication
}

Malaria is the most important serious Anopleles-borne protozoan disease in 2017 , there were an estimated 219 million cases of malaria in 90 countries with deaths reached 435000. WHO African Region carries a disproportionately high share of the global malaria burden. In 2017, the region was home to $92 \%$ of malaria cases and $93 \%$ of deaths. Total funding for control and elimination reached an estimated US\$ 3.1 billion. Contributions of endemic countries governents amounted to US\$ 900 million, about $28 \%$ of the total funding (WHO, 2018).

That's why it is the highly funded disease for researches that includes prophylactic measures, vaccinations trials, and recent treatment medications up-dates. Mortality ratehence-had fell dramatically lower for the past few years but still tops other potentially risky diseases.

Some population groups are at higher risk of contracting malaria, and developing severe disease, than others. These include infants, children under 5 years of age, pregnant women and HIV/AIDS patients, as well as non-immune migrants, mobile populations and travellers. National malaria control programmes need to take special measures to protect these population groups from malaria infection, taking into consideration their specific circumstances. Malaria is on top of the differential diagnosis of fever in a returning traveler especially from sub-Saharan Africa with infectious diseases as Yellow fever, Dengue fever and other viral hemorrhagic fevers as Lassa, Rift Valley, Ebola, Marburg, Crimean-Congo hemorhagic, chikunguunya, also African trypanosomiasis, leishmanaisis, loasis, onchocercaisis, typhus fever. As well as, other fevers caused by food-borne and water diseases by returning travelers are differentially diagnosed: travelers' diarrhea, cholera, typhoid, hepatitis $\mathrm{A} \& \mathrm{E}$, schistosomiasis, paragonimiasis, druncuncliasis, echinoccocosis and poliomyelitis. Others are meningococcal diseases,
HIV, hepatitis B \& C, plague, as well as sexually transmitted diseases, rabies and TB.

Clinically, malaria is suspected in the setting of any febrile illness after staying in a malaria endemic area or even as an airplanetransit with: tachycardia, tachypnea, chills, malaise, fatigue, diaphoresis, headache, cough, anorexia, nausea, vomiting, abdominal pain, diarrhea, arthralgia and/or myalgias. Physical findings may include: jaundice, splenomegaly and/or hepatomegaly. Severe complicated disease warrants mixed infection with $P$. falciparum including haemodynamic instability, pulmonary edema, severe anemia, massive intravascular hemolysis, coagulopathy, hypoglycemia, metabolic acidosis, renal-failure; hepatic dysfunction, altered mental status, focal neurological deficits and seizures. The four usually non-malignant human types are $P$. malariae, $P$. vivax, $P$. ova$l e$, and $P$. Knowlesi. The potentially fatal $P$. falciparum does not have liver hypnozoites responsible for relapses as in others. Asymptomatic subclinical cases in endemic areas usually require no treatment except falciparum one (El-Bahnasawy et al, 2010). However, $P$. vivax still an important cause of morbidity and mortality across the Americas, Horn of Africa, East and South East Asia. Control of transmission is hampered by emergence of chloroquine resistance and several intrinsic characteristics of infection includeing asymptomatic carriage, challenges with diagnosis, difficulty to eradicate the carrier state and early gametocyte appearance (Martin and Vinetz, 2018).

Prophylaxis starts here with environmental friend anti-mosquito repellent(s) side by side with chemoprophyaxis before travelling to endemic areas, and continued regularly during stay there \& following departure from endemic area to eradicate stages released from liver. These include chloroquine sensitive malaria, and also chloroquine resistant malaria with another options of atovaquone/proguanil (short term travelers pre- 
fers although expensive), mefloquine (long term travelers prefers as weekly dosing) and also doxycycline. Chemoprophylaxis agents vary with respect to cost, adverse effects and dosing schedule.

Chloroquine formulations include chloroquine phosphate and hydroxycholoroquine, mainly a blood schizonticide given orally once weekly starting a week prior to travel once weekly while in malarial endemic area and then once weekly for four weeks after being back. Atovaquine/proguanil (acting synergistically) targets liver schizonts given orally beginning two days prior to traveling, during exposure and for a week after returning back. Mefloquine is a blood schizonticide given orally weekly beginning two weeks prior to traveling, during exposure and four weeks after being back .Doxycycline is also a blood schizonticide given orally daily one to two days prior to travelling, daily during exposure, and daily for four weeks after being back. But, antimalarial drugs taken for prophylaxis by travelers can delay appearance of malaria symptoms by weeks or months, long after he left the malaria-endemic area (particularly with $P$. vivax and $P$. ovale, both of which can produce dormant liver stage parasites; the liver stages may reactivate and cause disease months after the infective mosquito bite (CDC, 2018).

Children must also receive antimalarial prophylaxis before travel to endemic areas in the form of chloroquine or mefloquine that are options for use in infants and children of all ages and weights. But, atovaquine/proguanil can be given to children more than $5 \mathrm{~kg}$. and doxycycline to those more than 8 years old.

Pregnant women are at risk for still-birth, abortion, and serious complications for both mother and fetus. They should be advised to defer travelling until after delivery, otherwise chloroquine or mefloquine can be given but doxycycline is contraindicated due to potential fetal dysplasia, inhibition of bone growth and dental discoloration.

Primaquine is used mainly as primary pro- phylaxis as it has schizonticidal activity in non-falciparum malaria and as relapse prevention to eradicate hypnozoites but not in G6PD (glucose 6 phosphate dehydrogenase) deficient patients and also not given in pregnancy for possibility of any fetal G6PD that can cause haemolytic anemia.

Drug-drug interactions of those medications with warfarin, anti-arrythmics, and immunosuppressants are common drugs for potential consideration reviewed before travel. Anti-mosquito measures include bite prevention, by mosquitoes-repellants on clothes \& bed nets as DEET, Picardin, or PMD.

Nursing roles: 1- Avoid treatment on empty stomach, must give the first dose under a doctor supervision and repeat dose in vomiting within 30 minutes. 2- Must submit a report; no improvement after $48 \mathrm{hrs}$ or deterioration and at once transfer patient to ICU. 3- Report any new symptom.

Overall, prophylactic measures must still need more reinforcement and collaborations of World Health Authorities with strict before travel counseling education for travelers to endemic areas to avoid spread \& contain this serious illness. Guidelines are changeably variable as of emergence of resistance strains and travelers must review those of the WHO and CDC guidelines before travel.

\section{References and Resources}

CDC, 2018: Morbidity and Mortality Weekly Report Surveillance Summaries Vol. 67/No. 7

El-Bahnasawy, MM, Dabbous HKh, Morsy, T A, 2010: Imported malaria as a threat to Egypt. J. Egypt. Soc. Parasitol. 40, 3:773-87

Martin, TCS, Vinetz, JM, 2018: Asymptomatic Plasmodium vivax parasitaemia in the lowtransmission setting: the role for a populationbased transmission-blocking vaccine for malaria elimination. Malar J. 17, 1:89

WHO, 2018: Guidelines for the treatment of malaria $\left(3^{\text {rd }}\right.$ edition $)$

We are:

TOSSON A. MORSY, morsyegypt2014@gmail.com, HAZEM H. M. KHALIL, Hazemkhali1952@gmail.com SOHA MAMDOUH I. NASSAR, sohamamdouh_nassar@yahoo.com 